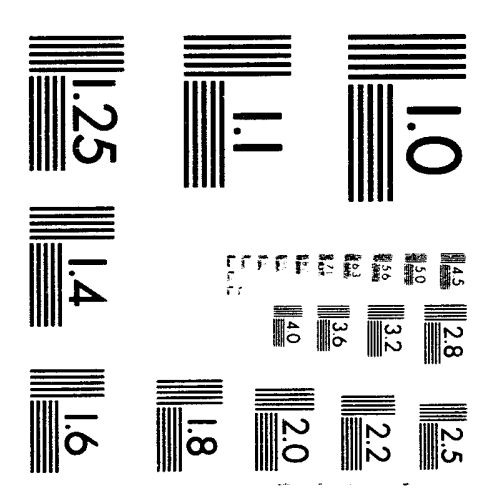



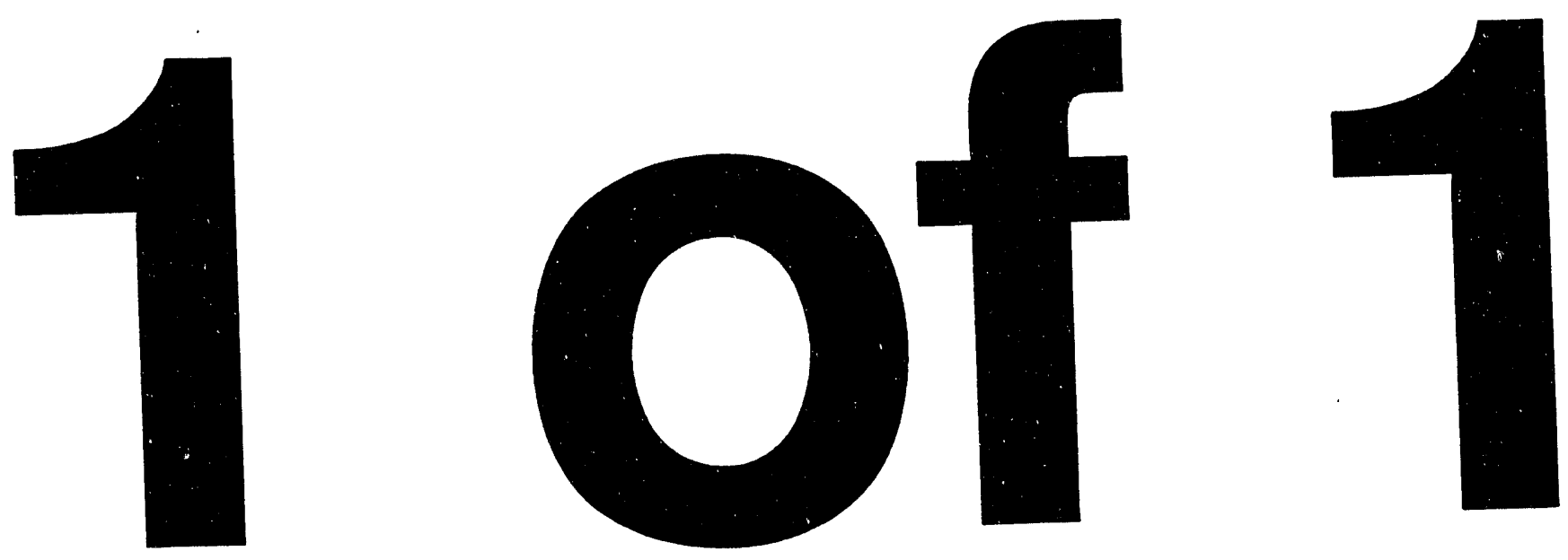
PPPL-2986

UC-427

COMPARISON OF INITIAL VALUE AND EIGENVALUE CODES

FOR KINETIC TOROIDAL PLASMA INSTABILITIES

BY

M. KOTSCHENREUTHER, G. REWOLDT AND W.M. TANG

APRIL, 1994

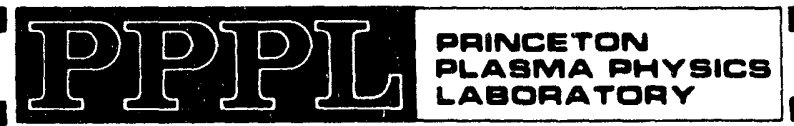

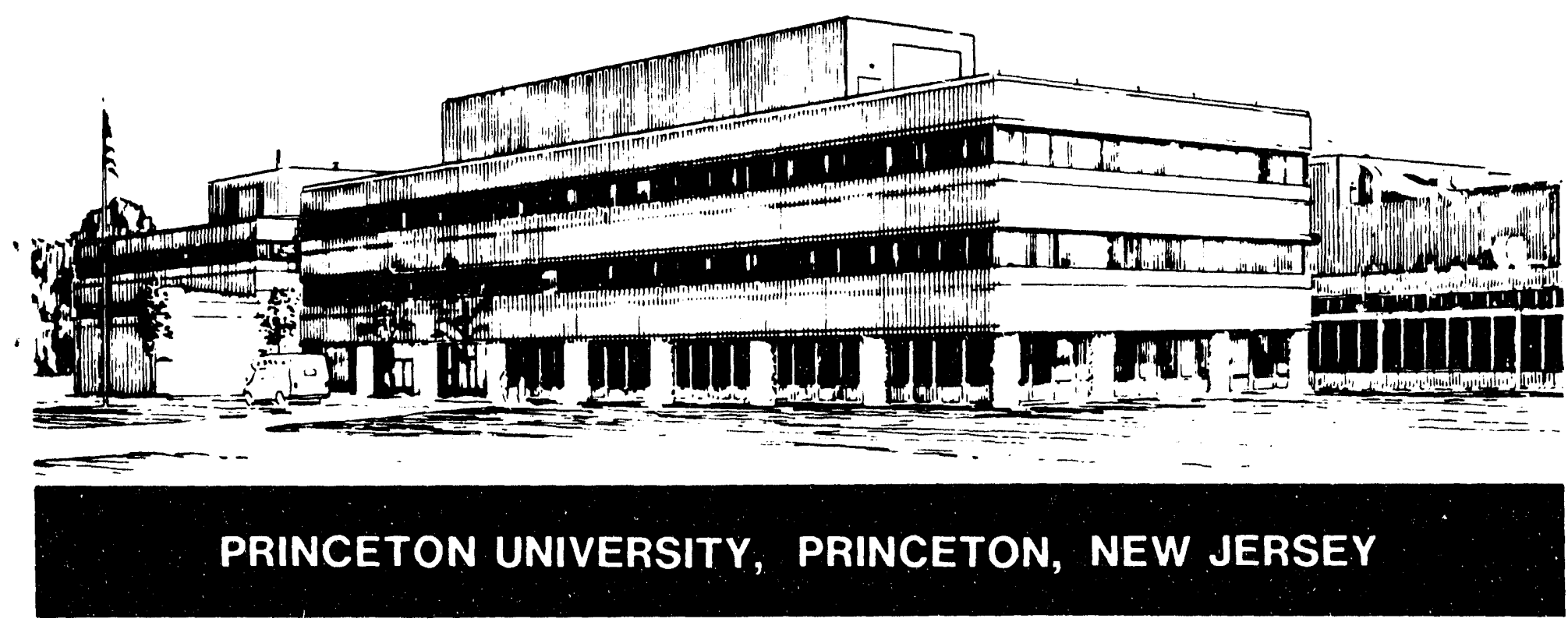




\section{NOTICE}

This report was prepared as an account of work sponsored by an agency of the United States Government. Neither the United States Government nor any agency thereof, nor any of their employees, makes any warranty, express or implied, or assumes any legal liability or responsibility for the accuracy, completeness, or usefulness of any information, apparatus, product, or process disclosed, or represents that its use would not infringe privately owned rights. Reference herein to any specific commercial produce, process, or service by trade name, trademark, manufacturer, or otherwise, does not necessarily constitute or imply its endorsement, recommendation, or favoring by the United States Government or any agency thereof. The views and opinions of authors expressed herein do not necessarily state or reflect those of the United States Government or any agency thereof.

\section{NOTICE}

This report has been reproduced from the best available copy.

Available in paper copy and microfiche.

Number of pages in this report: 25

DOE and DOE contractors can obtain copies of this report from:

Office of Scientific and Technical Information

P.O. Box 62

Oak Ridge, TN 37831;

(615) $576-8401$.

This report is publicly available from the:

National Technical Information Service

Department of Commerce

5285 Port Royal Road

Springfield, Virginia 22161

(703) $487-4650$ 


\title{
Comparison of Initial Value and Eigenvalue Codes for Kinetic Toroidal Plasma Instabilities
}

\author{
Mike Kotschenreuther \\ Institute for Fusion Studies \\ The University of Texas at Austin \\ Austin, Texas 78712 USA \\ G. Rewoldt and W.M. Tang \\ Plasma Physics Laboratory, Princeton University \\ P.O. Box 451, Princeton, New Jersey 08543-0451 USA
}

\begin{abstract}
In plasma physics, linear instability calculations can be implemented either as initial value calculations or as eigenvalue calculations. Here, comparisons between comprehensive linear gyrokinetic calculations employing the ballooniug formalism for high- $n$ (toroidal mode number) toroidal instabilities are described. One code implements an initial value calculation on a grid using a Lorentz collision operator and the other implements an eigenvalue calculation with basis functions using a Krook collision operator. An electrostatic test case with artificial parameters for the toroidal drift mode destabilized by the combined effects of trapped particles and an ion temperature gradient has been carefully analyzed both in the collisionless limit and with varying collisionality. Good agreement is found. Results from applied studies using parameters from the Tokamak Fusion Test Reactor (TFTR) experiment are also compared.
\end{abstract}




\section{Introduction}

Low frequency drift-type microinstabilities driven by ion temperature gradient and/or trapped-particle dynamics have continued to be strongly supported candidates to account for the anomalous transport observed in all tokamak plasmas. In the linear regime for these high- $n$ (toroidal mode number) toroidal instabilities, the equations to be solved reduce to onedimensional equations (in coordinate space) along the equilibrium magnetic field lines on a single, chosen magnetic surface, by means of the so-called ballooning representation. These are the linearized gyrokinetic equation, including an appropriate term to account for collisions, along with the quasineutrality condition and, in the electromagnetic case, Ampere's law or equivalent equations. These linear equations can be solved either by an initial value approach $\mathrm{s}$ by an eigenvalue approach for the mode eigenfrequency. In the present paper, comparisons are made between two completely independent computer codes employing these two different approaches, but solving essentially the same equations, enbodying the same underlying physics. As will be seen, the numerical results agree quite well for this instability in a variety of cases. One code implements an initial value approach on a grid using a Lorentz collision operator, and has been very briefly described in ref. [1]. The ccher code implements an eigenvalue approach employing basis functions and using a modified Krook collision operator, and has been described in detail in ref. [2]. For the present comparison, both of these calculations employ the same magnetic geometry, as described by the MHD equilibrium. This is the so-called "s- $\alpha$ " model MHD equilibrium [3] which corresponds to circular but non-concentric (due to the Shafranov shift) magnetic surfaces in toroidal geometry. Accordingly, effects due to magnetically trapped, as well as untrapped, electrons and ions are included in both calculations. Also, full finite Larmor radius effects for the ions are included in both calculations. For the present comparison, the equilibrium distribution functions of all species are taken to be Maxwellian. In accordance with the ballooning representation, both codes use ballooning coordinates. Both codes can include fully gyrokinetic ion, electron, impurity, and beam species. Both codes fully include kinetic effects from trapped particles and toroidal magnetic drifts. However, the algorithms used in the two codes are totally different. In particular, the initial value code solves for the distribution functions using finite differences in time and poloidal angle with a fully implicit scheme that is uncondition- 
ally stable, while the eigenvalue code uses an analytic integral solution for the distribution functions, and then numerically integrates over velocity space. The initial value code uses a Lorentz collision operator for pitch angle diffusion, while the eigenvalue code uses a modified Krook collision operator. The initial value code uses a grid in poloidal angle, while the eigenvalue code expands the eigenfunction in Hermite basis functions.

In section 2, the equations that constitute the starting point for both calculations are presented. Then, in section 3 , the initial value calculation is described in some detail, and in section 4 the eigenvalue calculation is described much more briefly, since considerable detail about it has already been given in ref. [2]. In section 5, results for an artificial test case are compared in the electrostatic limit, and in particular the behavior of the Lorentz and Krook collision models are compared. In section 6, results are compared for two realistic cases for the Tokamak Fusion Test Reactor (TFTR) both in the electrostatic limit and in the more general electromagnetic case. Finally, conclusions are presented in section 7 .

\section{Starting Equations}

The usual $r, \theta, \zeta$, toroidal coordinate system is used, with $r$ being the minor radius, $\theta$ the poloidal angle, and $\zeta$ the toroidal angle. The equilibrium magnetic field strength is $B=B_{0} / h(\theta)$, where $h(\theta)=1+\epsilon \cos (\theta), \epsilon=r / R$, and $R$ is the major radius of the center of the selected magnetic surface. Employing the ballooning representation, the perturbed electrostatic potential is expressed in the form

$$
\tilde{\Phi}(r, \theta, \zeta, t)=\exp [i n \zeta-i n q(r) \theta] \sum_{p=-\infty}^{\infty} \hat{\phi}(\theta-2 \pi p, r, t) \exp [i n q(r) 2 \pi p] .
$$

Note that $\tilde{\Phi}$ is periodic in the toroidal and poloidal angles, while $\hat{\phi}$ is defined in the infinite domain in $\theta$ and is not periodic. Similar forms apply for the perturbed distribution function $\tilde{f}_{s}$ for species $s$, and for $\tilde{A}_{\|}$, the component of the perturbed vector potential parallel to the equilibrium magnetic field. For the present comparison, $\tilde{A}_{\perp}$, the perpendicular component of the perturbed vector potential, is neglected. 
With these definitions, the linearized gyrokinetic equation in the ballooning representation using the " $\mathrm{s}-\alpha$ " model MHD equilibrium is

$$
\frac{\partial}{\partial t} \hat{g}_{s}+\frac{v_{\|}}{q R} \frac{\partial}{\partial \theta} \hat{g}_{s}+i \omega_{d s} \hat{g}_{s}+C\left(\hat{g}_{s}\right)=\frac{e_{s}}{T_{s}} F_{m s} J_{0}\left(\frac{\partial}{\partial t}+i \omega_{* s}^{T}\right)\left[\hat{\phi}(\theta)-\frac{v_{\|}}{c} \hat{A}_{\|}(\theta)\right],
$$

where $\hat{g}_{s} \equiv \hat{f}_{s}+\left(e_{s} / T_{s}\right) F_{m s} \hat{\phi}(\theta), \omega_{d s}=\omega_{* s}\left(L_{n s} / R\right)\left(E / T_{s}\right)\left(1+v_{\|}^{2} / v^{2}\right)\{\cos \theta+$ $[\hat{s} \theta-\alpha \sin \theta] \sin \theta\}, k_{\theta}=-n q / r, k_{\perp}=k_{\theta}\left\{1+[\hat{s} \theta-\alpha \sin \theta]^{2}\right\}^{1 / 2}, \hat{s} \equiv$ $(r / q)(d q / d r), \alpha \equiv-q^{2} R(d \beta / d r), \omega_{* s}^{T} \equiv \omega_{* s}\left\{\left[1+\eta_{s}\left[\left(E / T_{s}\right)-3 / 2\right]\right\}, J_{0} \equiv\right.$ $J_{0}\left(k_{\perp} v_{\perp} / \Omega_{s}\right)$, and the rest of the notation is standard.

The collision operator used in the initial value code is a Lorentz operator:

$$
C\left(\hat{g}_{e}\right)=\nu_{e}(E) \frac{\partial}{\partial \xi}\left(1-\xi^{2}\right) \frac{\partial}{\partial \xi} \hat{g}_{c}
$$

and $C\left(\hat{g}_{i}\right)=0$, where $\xi \equiv v_{\|} / v, \nu_{e}(E) \equiv\left[\nu_{e i} /\left(v / v_{e}\right)^{3}\right]\left[Z_{f}+H_{c e}\left(v / v_{c}\right)\right]$, $v_{e} \equiv\left(2 T_{e} / m_{\epsilon}\right)^{1 / 2}, Z_{f} \equiv \sum_{i} Z_{i}^{2} n_{i} / n_{e}, H_{e e}(x) \equiv \exp \left(-x^{2}\right) /(\sqrt{\pi} x)+[1-$ $\left.1 /\left(2 x^{2}\right)\right] \operatorname{erf}(x)$, and $\nu_{e i} \equiv 4 \pi n_{e} e^{2} \ln \lambda /\left[\left(2 T_{\epsilon}\right)^{3 / 2} m_{e}^{1 / 2}\right]$. This form of the Lorentz operator conserves particle number and energy, but not momentum.

In the eigenvalue code, on the other hand, a modified "Krook" collision operator is used:

$$
C\left(\hat{g}_{e}\right)=-\nu_{f e}(E, \Lambda) \hat{h}_{e}
$$

for trapped electrons,

$$
C\left(\hat{g}_{e}\right)=F_{m e}\left[\int_{\text {trapped }} d^{3} v \nu_{f e}(E, \Lambda) \hat{h}_{e}\right]\left[\int_{\text {untrapped }} d^{3} v F_{m e}\right]^{-1}
$$

for untrapped electrons, and (for this comparison) $C\left(\hat{g}_{i}\right)=0$ for ions, where $\hat{h}_{s} \equiv \hat{g}_{s}+\left(e_{s} / T_{s}\right) F_{m s}\left(1-\omega_{* s}^{T} / \omega\right) J_{0} \hat{\psi}_{\|} \exp \left[-i\left(k_{\perp} v_{\perp} / \Omega_{s}\right) \sin \chi\right], \chi$ is the gyrophase, $\hat{\psi}_{\|} \equiv(i \omega q R / 2 c)\left[\int_{-\infty}^{\theta} d \theta^{\prime} \hat{A}_{\|}\left(\theta^{\prime}\right)-\int_{\theta}^{\infty} d \theta^{\prime} \hat{A}_{\|}\left(\theta^{\prime}\right)\right], \Lambda \equiv \mu B_{0} / E$, and

$$
\nu_{f e}(E, \Lambda)=\frac{\nu_{e}(E)(r / R)}{|(1-r / R)-\Lambda|^{2}} \frac{0.111 \delta+1.31}{11.79 \delta+1}
$$

In the "old" Krook operator $\delta=\delta_{\text {old }} \equiv\left[|\omega| /\left(\nu_{e i} Z_{f}\right)\right]$, and in the "new" Krook operator $\delta=\delta_{\text {new }} \equiv\left[|\omega| /\left(\nu_{e i} Z_{f} \times 37.2 R / r\right)\right]^{1 / 3}$. This modified Krook operator is constructed so as to conserve particle number, but not momentum or energy. 


\section{Initial Value Calculation}

In this section the algorithm for the initial value code is described. The algorithm is unique among algorithms for solving the gyrokinetic equation in that it is fully implicit, and has no time step restrictions on stability. Implicitness arises in two parts: the finite difference scheme for computing the distribution function and the field solution procedure. As a result, the code time step is not restricted by the Courant Freidricks Levy criterion for either single particle motion or collective wave motion, e.g. electron motion or Alfvén waves. The time step is only restricted by accuracy requirements to be significantly shorter than the mode frequency of interest. This allows much longer time steps which save about two orders of magnitude of computer time when full electron dynamics are included. Also, the algorithm is unique in that it uses a diffusive pitch angle scattering (Lorentz) collision operator, rather than a Krook operator. Because it is a diffusion operator like the actual collision operator, and because pitch angle diffusion is usually the dominant diffusion process for typical collision frequency regimes, the physics associated with sharp boundaries in velocity space is automatically treated correctly both qualitatively and quantitatively. Such sharply varying behavior in velocity space arises at the trapped/untrapped boundary and sometimes near Landau resonances.

\subsection{Coordinates and equations}

It is found to be computationally convenient to remove the time derivative term proportional to $\partial \phi / \partial t$. For this, we define a quantity $f \equiv g-e J_{0} F_{m} \phi / T$, which satisfies

$$
\begin{aligned}
\frac{\partial f}{\partial t} & +i \omega_{d} f+\frac{v_{\|}}{q R} \frac{\partial}{\partial \theta} f+C(f)=\frac{e}{T} F_{m}\left\{v_{\|}\left[\frac{1}{q R} \frac{\partial\left(J_{0} \phi\right)}{\partial \theta}-\frac{J_{0}}{c} \frac{\partial A_{\|}}{\partial t}\right]\right. \\
& \left.+i \omega_{*}^{T} J_{0}\left[\phi-\frac{v_{\|}}{c} A_{\|}\right]-i \omega_{d} J_{0} \phi\right\},
\end{aligned}
$$

where we have temporarily omitted the hats $\left(^{\wedge}\right)$ and the species subscripts.

The coordinates used are the energy $E$, the pitch angle variable $\lambda \equiv$ $2 \mu / E \equiv v_{\perp}^{2} / v^{2} B(\theta)$, and the ballooning coordinate $\theta$. For each $\lambda$ and $E$, 
there are two different signs of the velocity $v_{\|}= \pm \sqrt{E(1-\lambda B(\theta))}$, so there is an additional coordinate $\sigma= \pm 1$ for the sign of $v_{\|}$.

The coordinates $\lambda$ and $E$ are merely parameters in the gyrokinetic equation, but they must be integrated over in order to obtan the perturbed density and currents for quasineutrality and Ampére's law. Velocity integrals in these coordinates have the form

$$
\int d \mathbf{v}=\frac{\pi}{2} B(\theta) \int d E \sqrt{E} d \lambda / \sqrt{1-\lambda B(\theta)}
$$

To minimize the number of grid points and thus computational effort, but nonetheless maintain good accuracy in these integrals, the grid points are chosen using Gaussian integration rules as much as possible.

Recall that there are both trapped and untrapped regions of velocity space. The untrapped region has $0 \leq \lambda \leq 1 / B_{\max }$, and the trapped region has $1 / B_{\max } \leq \lambda \leq 1 / B_{\min }$, where $B_{\max }$ and $B_{\min }$ are the maximum and minimum values of $B(\theta)$ for $0 \leq \theta \leq 2 \pi$. The distribution function often behaves differently in these two regions, so the coordinate grid is different. For the untrapped region, the $\lambda$ coordinates are modified to remove the singularity in the denominator in eq. (8). In the untrapped region, new coordinates $X$ are chosen, with

$$
X=\sqrt{1-B_{\max } \lambda} .
$$

Gaussian integration rules are used in the untrapped region in the variable $X$ to evaluate integrals such as eq. (8). The Gaussian integration rules specify the values of $X$ and thus the $\lambda$ grid points. These coordinates remove the singularity at the separatrix in eq. (8). These coordinates are also more closely spaced near the separatrix between the trapped and untrapped boundary, which is desirable since this is a transition region where the distribution function often varies rapidly.

In the trapped region, the $\lambda$ grid is chosen so that the points where $v_{\|}=0$ corresponds to grid points in $\lambda$. Specifically, given the $\theta$ grid points $\theta_{i}$ for $-\pi \leq \theta \leq \pi$, the $\lambda$ grid points $\lambda_{i}$ are chosen so that

$$
1-\lambda_{i} B\left(\theta_{i}\right)=0 \text {. }
$$

This choice insures that the mirror points where $v_{\|}=0$ always lie exactly on a gridpoint. There are important boundary conditions at these points, 
as discussed below. Those conditions are easiest to apply numerically if the mirror points are at a gridpoint. In addition, this choice also automatically increases the density of grid points near the separatrix between the trapped and untrapped region.

The $\theta$ grids are chosen to be equally spaced, with typically 24 to 36 grids per $2 \pi$ interval in $\theta$.

Gaussian integration rules are used in the $E$ variable. The interval from 0 to $\infty$ is broken into two parts. From 0 to $E_{\text {cut }}$, Gaussian integration rules of order $N_{1}$ are used with Gaussian weight factor 1 . For $E_{\text {cut }}$ to $\infty$, integration rules of order $N_{2}$ with weight factor $e^{-E}$ are used. After some experimentation, choices which are usually highly accurate with few grid points have $E_{\text {cut }} \sim 2-3,3 \leq N_{1} \leq 8$, and $2 \leq N_{2} \leq 3$.

\subsection{Boundary conditions}

The boundary conditions are standard for the gyrokinetic equation in ballooning coordinates. The distribution function must vanish as $\theta \rightarrow \pm \infty$. The gyrokinetic equation is first order in $\theta$, so boundary conditions can only be enforced at one point in $\theta$. The physical way to do this in the code is to set $f=0$ at the rightmost $\theta$ grid for $v_{\|}<0$ and set $f=0$ at the leftmost $\theta$ grid for $v_{\|}>0$. This is chosen because if $f=0$ for all $\theta$ values outside of the last grid points, then the distribution with $v_{\|}<0$ which convects into the simulation region from the right would have $f=0$ at the rightmost grid, and similarly for the distribution with $v_{\|}>0$ and the leftmost grid point. These boundary conditions are also found in practice to result in physically well behaved solutions.

Separate trapped particle regions exist every $2 \pi$ in $\theta$. For fixed $\lambda$ and $E$, these regions have a limited extent in $\theta$, and end at the mirror points where $v_{\|}=0$. The boundary conditions at the mirror points are the usual ones, namely that the distribution function for $v_{\|}<0$ must equal the distribution function for $v_{\|}>0$ at the $\theta$ value of the mirror point.

For equilibrium geometry with up-down symmetry (such as the one used in this paper), symmetric eigenfunctions exist. Even parity modes have $f(\theta, \sigma=+1)=f(-\theta, \sigma=-1)$ and $\phi(\theta)=\phi(-\theta)$, and odd parity modes have $f(\theta, \sigma=+1)=-f(-\theta, \sigma=-1)$ and $\phi(\theta)=-\phi(-\theta)$. For such cases, the computational effort can be cut in half since only $\theta \geq 0$ need be simu- 
lated.

\subsection{Finite difference scheme}

The implicit scheme to allow large time steps is a vital ingredient of the utility of this numerical scheme. Implicitness arises in two parts in this algorithm: the finite difference scheme for computing the distribution function and the field solution algorithm. In this section the implicit finite difference scheme for computing the distribution function is explained. For full electron dynamics, this allows a time step about two orders of magnitude larger than would otherwise be possible.

The collisionless gyrokinetic equation is finite differenced first. The diffusive collision operator is applied in a second step (as in a splitting scheme).

The collisionless gyrokinetic equation is a first order partial differential equation with convection in the $\theta$ direction. Though many finite difference schemes exist for such equations, the scheme presented here is unique (to the authors' knowledge) in that it is 1) implicit and unconditionally stable 2) computationally almost as fast as an explicit scheme, 3) second order accurate in space and 4) can be either first or second order accurate in time, depending on the amount of implicitness desired.

The second order accuracy in space arises from being space centered. The scheme allows variable time centering, with a parameter $\delta$ which varies from 0 (fully explicit) to 1 (fully implicit), and $\delta=1 / 2$ corresponds to a time centered, second order accurate scheme. (Typically $\delta=0.6$ is chosen.) The following terms in the gyrokinetic equation are finite differenced as shown:

$$
\begin{aligned}
& \omega_{d} f \rightarrow \frac{\omega_{d}}{2}\left[(1-\delta)\left(f_{i}^{n}+f_{i+1}^{n}\right)+\delta\left(f_{i}^{n+1}+f_{i+1}^{n+1}\right)\right] \\
& \frac{\partial f}{\partial t} \rightarrow \frac{1}{2}\left[\frac{\left(f_{i}^{n+1}-f_{i}^{n}\right)}{\Delta t}+\frac{\left.f_{i+1}^{n+1}-f_{i+1}^{n}\right]}{\Delta t}\right] \\
& \frac{\partial f}{\partial \theta} \rightarrow \delta \frac{\left(f_{i+1}^{n+1}-f_{i}^{n+1}\right)}{\Delta \theta}+(1-\delta) \frac{\left(f_{i+1}^{n}-f_{i}^{n}\right)}{\Delta \theta} .
\end{aligned}
$$

Here, $n$ labels the time step and $i$ labels the $\theta$ grid point.

The finite diffcrencing of the $\phi$ and $A_{\|}$terms uses exactly the same scheme as the distribution function $f$ above with the same value of $\delta$. This leads to 
a finite difference equation for eq. (7) which is of the form

$$
\begin{aligned}
& D_{1} \ddot{\prime}^{n}+D_{2} f_{i+1}^{n}+D_{3} f_{i}^{n+1}+D_{4} f_{i+1}^{n+1}=F_{1} \phi_{i}^{n}+F_{2} \phi_{i+1}^{n} \\
& +F_{3} \phi_{i}^{n+1}+F_{4} \phi_{i+1}^{n+1}+F_{5} A_{\|_{1}}^{n}+F_{6} A_{\|_{1+1}}^{n}+F_{7} A_{\|_{1}}^{n+1}+F_{8} A_{\|_{1}+1}^{n+1},
\end{aligned}
$$

where tive coefficients are functions of $\lambda, E$, and $\theta_{i}$.

For given $\phi$ and $A_{\|}$this difference scheme is stable for all time steps if $\delta \geq 1 / 2$. For constant $v_{\|}$, it is straightforward to allalyze the stability for a perturbation $f \sim e^{i k \theta}$. The numerical stability growth factor after one time step is less than or equal to one for all $k$ and all time steps if $\delta \geq 1 / 2$. From experience, eq. (14) is also found to always be stable for $\delta>1 / 2$ when $v_{\|}$is a function of $\theta$.

It is straightforward to advance eq. (14) by one time step given $\phi$ and $A_{\|}$. If $f_{i}^{n+1}$ is known, eq. (14) can be used to compute $f_{i+1}^{n+1}$. Recall that the boundary conditions for the untrapped region are that $f=0$ at the rightmost $\theta$ grid for $v_{\|}<0$ and $f=0$ at the leftmost $\theta$ grid for $v_{\|}>0$. To solve eq. (14), start at the boundary where $f=0$. The adjacent value of $f$ in the simulation region is computed by solving eq. (14) at the boundary, and then consecutively applying eq. (14) for adjacent values of $i$ till $f_{i}^{n+1}$ is known for all $i$ values.

For the trapped particle regions the procedure is similar, except that the boundary conditions do not specify a particular value of $f_{i}^{n+1}$ at any $i$, but only that the values at the mirror points must be the same for $\sigma= \pm 1$. Therefore, both an inhomogeneous and a homogeneous solution to eq. (14) is computed for each separate trapped region, and the appropriate linear combination in each region is used for $f$. Specifically, eq. (14) i's solved twice, once with $f_{i}^{n+1}=0$ at one mirror point (the one with largest $\theta$ ), and once with $f_{i}^{n+1}=1$ at that mirror point and also $\phi=A_{\|}=0$ assumed. Both solutions are computed as above for $\sigma=-1$ until the other mirror point is reached. Then, for both solutions, $f_{i}^{n+1}$ at that mirror point for $\sigma=1$ is set equal to the value at $\sigma=-1$. Thus both solutions automatically solve the boundary conditions at this point, so any linear combination will also. Consecutive application of eq. (14) to both homogeneous and inhomogeneous solutions until the first mirror point is reached gives $f_{i}^{n+1}$ for all $i$ for $\sigma=+1$. Then the appropriate linear combination of solutions is used so that $f_{i}^{n+1}$ is equal at the first mirror point for $\sigma= \pm 1$. 
For symmetric modes satisfying $f(\theta, \sigma)=f(-\theta,-\sigma)$ the procedure is almost the same, except that only half the $\theta$ values are used. When consecutively applying eq. (14) for $\theta \geq 0$ and $\sigma=-1$, eventually $\theta=0$ is reached. At that point $f(\theta, \sigma)=f(\theta,-\sigma)$ is used. Consecutive application of eq. (14) then proceeds for $\sigma=+1, \theta>0$.

\subsection{Collision operator}

The collision operator is a pitch angle scattering operator of the form

$$
C(f)=\nu_{e}(E) \frac{\partial}{\partial \xi}\left(1-\xi^{2}\right) \frac{\partial}{\partial \xi} f
$$

Time advancing the gyrokinetic equation one full time step is a two-step process. First, the collisionless gyrokinetic equation is advanced one time step according to eq. (14). The result of this then has the collision operator applied to it as follows

$$
f^{n+1}-\Delta t C\left(f^{n+1}\right)=f_{\text {collisionless }}^{n+1}
$$

After this equation is solved, the distribution has been fully advanced one step. For this two step procedure to be accurate, the time step must be small compared to the collision frequency. This requirement is usually easy to satisfy for plasma parameters over most of the plasma volume for modern fusion experiments. However, even for large time steps eq. (16) is still stable.

The collision operator is finite differenced in a standard way, namely

$$
\begin{aligned}
& \frac{\partial}{\partial \xi}\left(1-\xi^{2}\right) \frac{\partial}{\partial \xi} \longrightarrow \frac{\Gamma_{\ell}-\Gamma_{\ell-1}}{\xi_{\ell}-\xi_{\ell-1}} \\
& \Gamma_{\ell}=\left(1-\xi_{\ell+1 / 2}^{2}\right) \frac{f_{\ell+1}-f_{\ell}}{\xi_{\ell+1}-\xi_{\ell}}
\end{aligned}
$$

where $\xi_{\ell}=\sqrt{1-\lambda_{\ell} B(\theta)}$ and $\xi_{\ell+1 / 2}=\left(\xi_{\ell}+\xi_{\ell+1}\right) / 2$.

The tridiagonal matrix inversion associated with eq. (17) is implemented in a way which vectorizes over the $\theta$ grid, which greatly speeds execution. (The $\lambda$ grid cannot be vectorized over because of the recursive nature of the solution procedure for tridiagonal matrices.) Since there are a different number of $\lambda$ grid points at each $\theta$ grid (due to the existence of the trapped particle 
regions), vectorization is facilitated by adding dummy matrix elements to the tridiagonal matrix so that all $\theta$ grid points have the same number of $\lambda$ grid points.

\subsection{Field solution and time-stepping procedures}

The calculation of the fields $\phi$ and $A_{\|}$is also fully implicit, which also makes this code unique. A plasma has many waves which are collective modes of oscillation due to the electromagnetic fields. If the fields were computed explicitly, the time step would be limited by the Courant-Freidricks-Levy criterion due to these waves, even though the code was fully implicit for the electron and ion motions in a given field. In the present case, the most limiting wave is a shear Alfvén wave along $\theta$. For the implicit field solution scheme here, accuracy is the only limit on time step, not stability. In practice, this allows time steps about an order of magnitude larger than for an explicit field solution scheme.

To implement the implicit scheme, it is first necessary to numerically compute response functions for the system before actual simulation begins. These response functions are conceptually the density and current response of the plasma to a perturbation of the fields which is a delta function in $\theta$. The response is computed for a delta function at each $\theta$ grid point. Since the coefficients of the gyrokinetic equation are time irdependent, the same response functions apply for the entire simulation time.

Specifically, the response functions are computed as follows. First the $\phi$ response is computed. The gyrokinetic equation is advanced one time step using eqs. (14) and (23) and the procedure in the subsection above, for a sequence of trial functions. In these trial functions, all fields are taken to be zero for each grid point except one where $\phi_{j}^{n+1}=1$. The distribution function $f^{n}$ is taken to be zero, and the distribution at $f^{n+1}$ is computed. Then the velocity integrals which are needed in quasineutrality and Ampére's law are computed, i.e. the density and current. Then a similar calculation is then done with trial functions where the only nonzero field has $A_{\|_{s}^{n+1}}^{n+1}=1$ at one grid point. These calculations are done individually for trial functions at every grid point.

Since any fields with $\phi^{n+1}$ and $A_{\|}^{n+1}$ can be written as a linear combination of the trial functions above, and since the gyrokinetic equation is linear, the 
density and current response to $\phi^{n+1}$ and $A_{\|}^{n+1}$ can be written as a linear combination of the response functions above. Thus, the current and density response due to $\phi^{n+1}$ and $A_{\|}^{n+1}$ can be written as matrices

$$
\begin{aligned}
& n_{i}=\sum_{j} M_{i j}^{1} \phi_{j}^{n+1}+M_{i j}^{2} A_{\| j}^{n+1} \\
& j_{i}=\sum_{j} M_{i j}^{3} \phi_{j}^{n+1}+M_{i j}^{4} A_{\| j}^{n+1} .
\end{aligned}
$$

These matrices are used in the following time-stepping procedure which advances $f$ from time step $n$ to time step $n+1$. Since eq. (14) is linear, the solution can be written as the sum of a homogeneous and an inhomogeneous part

$$
f=f_{1}+f_{2}
$$

where

$$
\begin{aligned}
D_{1} f_{1_{i+1}}^{* n}+D_{2} f_{1_{i+1}}^{* n}+D_{3} f_{1_{i+1}}^{* n+1}+D_{4} f_{1_{i+1}}^{* n+1}= & F_{1} \phi_{i}^{n}+F_{2} \phi_{i+1}^{n} \\
& +F_{5} A_{i}^{n}+F_{6} A_{i+1}^{n} \\
f_{1}^{n+1}-\Delta t \mathcal{C}\left(f_{1}^{n+1}\right)= & f_{1_{i+1}}^{* n+1}
\end{aligned}
$$

and $f_{1}^{n}=f^{n}$, and

$$
\begin{aligned}
D_{1} f_{2 i}^{* n}+D_{2} f_{2 i+1}^{* n}+D_{3} f_{2 i}^{* n+1}+D_{4} f_{i+1}^{* n+1}= & F_{3} \phi_{i}^{n+1}+F_{4} \phi_{i+1}^{n+1} \\
& +F_{7} A_{i}^{n+1}+F_{8} A_{i+1}^{n+1} \\
f_{2}^{n+1}-\Delta t \mathcal{C}\left(f_{2}^{n+1}\right)= & f_{2}^{* n+1}
\end{aligned}
$$

where $f_{2}^{n}=0$.

The inhomogeneous solution $f_{1}$ is computed as in the section on the timestepping scheme, since the right-hand side of eq. (20) is known after the $n$th step. However, the right-hand side of eq. (21) is not known, since $\phi^{n+1}$ and $A_{\|}^{n+1}$ have yet to be computed. To find $\phi^{n+1}$ and $A_{\|}^{n+1}$, we use eq. (21) to write

$$
\begin{aligned}
& \sum_{s} n_{0 s} e_{s} \int d \mathbf{v} J_{0} f_{s}=n_{1}+n_{2} \\
& \sum_{s} n_{0 s} e_{s} \int d \mathbf{v} J_{0} v_{\|} f_{s}=j_{1}+j_{2}
\end{aligned}
$$


where $n_{1}$ and $n_{2}$ are the density contributions from $f_{1}$ and $f_{2}$, respectively, and similarly for $j_{1}$ and $j_{2}$. Having solved eq. (20), $n_{1}$ and $j_{1}$ are easily computed. The response function matrices eq. (20) can be used to substitute for $n_{2}$ and $j_{2}$, to obtain from quasineutrality and Ampére's law

$$
\begin{aligned}
& \sum_{j} n_{U i}\left(1-\Gamma_{0}\right) e_{i} \frac{\phi_{i}^{n+1}}{T_{i}}=n_{1 i}+\sum_{j}\left(M_{i j}^{1} \phi_{j}^{n+1}+M_{i j}^{2} A_{\|}^{n+1}\right) \\
& \frac{c}{4 \pi}\left[1+(\hat{s} \theta-\alpha \sin \theta)^{2}\right] A_{i}^{n+1}=j_{1 i}+\sum_{j}\left(M_{i j}^{3} \phi_{j}^{n+1}+M_{i j}^{4} A_{\| j}^{n+1}\right) .
\end{aligned}
$$

These matrix equations are solved for the new fields $\phi^{n+1}$ and $A_{\|}^{n+1}$. Each dimension of the matrices is twice the number of $\theta$ grid points, and they are typically in the $200 \times 200$ range. In practice it is found that they are not problematic to invert in single precision on a CRAY. This is probably due to the fact that the largest elements in the matrix are concentrated near the diagonal, since the physical response of the system is greatest near the perturbing trial function. The inversion typically takes considerably less computation time than advancing the distribution function with eq. (14).

Having computed $\phi^{n+1}$ and $A_{\|}^{n+1}$, eq. (21) may now be solved, and thus $f^{n+1}$ obtained from eq. (19). With this, the equation has been fully advanced to the $n+1$ time step, and the same procedure is used to continue advancing the simulation in time.

\section{Eigenvalue Calculation}

The eigenvalue calculation has been described in some detail in refs. [2] and [4], so only a general outline of the procedure will be given here. Taking all perturbed quantities to have an $\exp (-i \omega t)$ time dependence and using the Krook collision operator given in eqs. (4) and (5), and changing from the dependent variable $\hat{A}_{\|}(\theta)$ to $\hat{\psi}_{\|}(\theta) \equiv(i \omega q R / 2 c)\left[\int_{-\infty}^{\theta} d \theta^{\prime} \hat{A}_{\|}\left(\theta^{\prime}\right)-\int_{\theta}^{\infty} d \theta^{\prime} \hat{A}_{\|}\left(\theta^{\prime}\right)\right]$, eq. (2) becomes a first-order ordinary differential equation in $\theta$. With the Krook collision operator, velocity-space variables enter only parametrically. This equation can be solved analytically, by separate procedures for trapped and untrapped particles as described in detail in ref. [4], to obtain exact solutions for $\hat{f}_{j}$ in terms of $\hat{\phi}$ and $\hat{\psi}_{\|}$. These solutions are then used in the quasineutrality condition $\sum_{s} e_{s} \int d^{3} v \hat{f}_{s}=0$. However, instead of using the 
component of Ampere's law parallel to the equilibrium magnetic field lines, the gyrokinetic moment equation, which comes from applying the operator $\sum_{s} e_{s} \int d^{3} v$ to the modified form of the gyrokinetic equation, is used. For the present comparison, $\hat{A}_{\perp}$ and the perpendicular component of Ampere's law, which are included in refs. [2] and [4], are omitted. The resulting system of two linear, homogeneous, non-Hermitian integrodifferential equations is solved by a Ritz method, where the unknown potentials are decomposed in terms of chosen basis functions

$$
\hat{\phi}(\theta)=\sum_{l=0}^{L-1} \hat{\phi}_{l} h_{l}(\theta)
$$

and correspondingly for $\hat{\psi}_{\| \mid}(\theta)$, where $h_{l}(\theta) \equiv H_{l}\left(\Delta^{1 / 2} \theta\right) \exp \left(-\Delta \theta^{2} / 2\right) M_{l}^{1 / 2}$, $H_{l}$ is the Hermite polynomial of order $l, M_{l} \equiv(\pi / \Delta)^{1 / 2} 2^{l} l !$, and $\Delta>0$ is an adjustable real parameter. Then, the operator $\left[T_{e} /\left(|e|^{2} n_{e}\right)\right] \int_{-\infty}^{\infty} d \theta h_{l^{\prime}}(\theta)$ is applied to each of the two equations, for $0 \leq l^{\prime} \leq L-1$. These operations have the effect of converting the two integrodifferential equations into a single large matrix equation,

$$
M(\omega)\left(\begin{array}{c}
\hat{\phi}_{1} \\
\vdots \\
\hat{\psi}_{\| 1} \\
\vdots
\end{array}\right)=\sum_{s} Z_{s}\left(\begin{array}{cc}
M_{l^{\prime} l}^{11} & M_{l^{\prime} l}^{s 12} \\
M_{l^{\prime} l}^{21} & M_{l^{\prime} l}^{* 22}
\end{array}\right)\left(\begin{array}{c}
\hat{\phi}_{1} \\
\vdots \\
\hat{\psi}_{\| 1} \\
\vdots
\end{array}\right)=\left(\begin{array}{c}
0 \\
\vdots
\end{array}\right) .
$$

Here, $M(\omega)$ is a $2 \times 2$ block matrix, with each block being an $L \times L$ matrix, where $L$ is the number of basis functions that are kept, $Z_{s} \equiv e_{s} /|e|$ is the charge number, and the detailed form of $M_{l^{\prime} l}^{s m^{\prime} m}(\omega)$ is given in ref. [2]. (In the electrostatic limit, the $\hat{\psi}_{\mid l l}$ are omitted and only the $M_{l^{\prime} l}^{s 11}$ block of $M$ is used.) The matrix $M(\omega)$ has $2 L$ eigenvalues $\lambda_{i}(i=1, \ldots, 2 L)$ and $2 L$ corresponding eigenvectors. Equation (25) can be satisfied only if $\operatorname{det}(M)=0$, and this can happen only if at least one of the $\lambda_{i}$ 's is zero, since $\operatorname{det}(M)=\prod_{i=1}^{2 L} \lambda_{i}$. Each of the $\lambda_{i}$ 's is a function of $\omega$. Thus, the eigenvalue condition on $\omega$ is $\lambda_{2}(\omega)=0$ for a single $\lambda_{i}$ corresponding to a chosen eigenvector (and eigenfunction). The eigenvalue of $\omega$ can be found by standard root-finding methods, such as Newton's method (method of false position). The number of basis functions, $L$, must be chosen sufficiently large that the eigenvalue $\omega$ is independent of $L$ to some acceptable accuracy, and is typically taken as $L=32$. 


\section{Artificial 'rest Case and Collision Models}

In this section, results of the two calculations are compared for an artificial test case in the electrostatic limit. The "s- $\alpha$ " model MHD equilibrium is used with $q=1.5, \hat{s} \equiv(r / q)(d q / d r)=1.0$, and $\alpha \equiv-q^{2} R(d \beta / d r)=0$, where $\beta \equiv\left(8 \pi / B_{0}^{2}\right) \sum_{s} n_{s} T_{s}$. The local plasma parameters, in standard notation, are $L_{n} / R=a / R=1 / 3, \eta_{i}=\eta_{e}=3.0, T_{i}=T_{\epsilon}=3.0 \mathrm{keV}, r / a=1 / 2$, $r / R=1 / 6, Z_{\text {eff }}=1.0$ (no impurities), $m_{i}=2$ a.m.u. (deuterium), $R=$ $240 \mathrm{~cm}, a=80 \mathrm{~cm}, r=40 \mathrm{~cm}$, and $L_{n}=80 \mathrm{~cm}$. First, in the collisionless limit, results for the real frequency $\omega_{r}$ and the growth rate $\gamma$ in units of the electron diamagnetic drift frequency $\omega_{* e}$ are shown in Fig. 1 for a range of $k_{\theta} \rho_{i}$ values, where $k_{\theta} \equiv-n q / r$ and $\rho_{i} \equiv\left(2 T_{i} / m_{i}\right)^{1 / 2} /\left(e B_{0} / m_{i} c\right)$. It is seen that the initial value results and the eigenvalue results for $\omega_{r}$ and $\gamma$ agree well over the whole range $0.03 \leq k_{\theta} \rho_{i} \leq 0.7$, with $|\Delta \omega| /|\omega| \lesssim 6 \%$.

The electron collision frequency is varied in Fig. 2 by varying the electron (and ion) density, since the electron collision frequency $\nu_{\epsilon}$ is approximately proportional to $n_{\mathrm{f}}=n_{i}$, while the ion collision frequency is still set to zero. Also, $k_{\theta} \rho_{i}$ is fixed at 0.5 . The results from the initial value and eigenvalue calculations for the departure of $\omega_{r}$ and $\gamma$ from their collisionless limits are shown as a function of $n_{e}$ in Fig. 2. The "old" form of the Krook collision operator given by eqs. (4) and (5), with $\delta=\delta_{\text {old }} \equiv\left[|\omega| /\left(\nu_{e i} Z_{f}\right)\right]$, is seen to give agreement for the growth rate with the Lorentz operator results from the initial value calculation in the small $n_{e}$ and large $n_{e}$ asymptotic limits, as it was constructed to do in ref. [2], but diverges rather badly in the transition region. However, changing only the coefficient and exponent in $\delta$, from $\delta=$ $\delta_{\text {old }} \equiv\left[|\omega| /\left(\nu_{e i} Z_{f}\right)\right]$ to $\delta=\delta_{\text {new }} \equiv\left[|\omega| /\left(\nu_{e i} Z_{f} \times 37.2 R / r\right)\right]^{1 / 3}$, brings the eigenvalue code Krook operator growth rate into good agreement with the initial value code Lorentz operator growth rate throughout the transition region as well. The agreement for the real frequency $\omega_{r}$ is improved at the same time for the lower density range, which is the more experimentally relevant range. However, obtaining accurate values for the growth rate was considered to be more important than for the real frequency, so that the coefficient and exponent in $\delta$ were chosen strictly to optimize the agreement of the growth rates. As will be seen in Sec. 6, the agreement for realistic experimental parameters of both the growth rate and the real frequency will be acceptable with $\delta=\delta_{\text {new }}$. 


\section{TFTR Experimental Cases}

In this section, two different TFTR L-mode experimental cases are considered. The first corresponds to TFTR shot 52504 at $r=37.9 \mathrm{~cm}$. The "s- $\alpha$ " model MHD equilibrium is used with $q=1.122, \hat{s}=0.9707$, and $\alpha=0.07612$. The global parameters are: $B_{0}=4.759 \mathrm{~T}, I_{p}=1.9774 \mathrm{MA}, a=79.88 \mathrm{~cm}$, $R=245.0 \mathrm{~cm}$, and $Z_{\text {fff }}=2.344$ (from a carbon impurity species with $s=C$ ). A hot deuterium beam species with $s=b$ is included in the calculations along with the background deuterium ion species with $s=i$. The local parameters at $r=37.9 \mathrm{~cm}$ are: $n_{c}=5.57 \times 10^{13} \mathrm{~cm}^{-3}, n_{b}=0.469 \times 10^{13} \mathrm{~cm}^{-3}$, $n_{C}=0.250 \times 10^{13} \mathrm{~cm}^{-3}, T_{c}=3.22 \mathrm{keV}, T_{i}=T_{C}=2.77 \mathrm{keV}, T_{b}=50$ $\mathrm{keV}, L_{n \epsilon}=L_{n C}=83.6 \mathrm{~cm}, L_{T e}=44.3 \mathrm{~cm}, L_{T i}=L_{T C}=37.3 \mathrm{~cm}, L_{S} \equiv$ $q R / s=283.2 \mathrm{~cm}, \eta_{e}=1.89, \eta_{i}^{\epsilon} \equiv\left(d \ln T_{i} / d r\right) /\left(d \ln n_{e} / d r\right)=2.24, \eta_{b}=0$, $r / R=0.155$, and $L_{n b}=64.5 \mathrm{~cm}$. The instability calculations are again performed in the electrostatic limit. The results from the initial value calculation and the eigenvalue calculation for $\omega_{r}$ and $\gamma$ are shown as a function of $k_{\theta} \rho_{\iota}$ in Fig. 3. Again, there is reasonable agreement, with $|\Delta \omega| /|\omega| \lesssim 12 \%$.

The second TFTR L-mode experimental case corresponds to TFTR shot 49982 at $r=38 \mathrm{~cm}$. The "s- $\alpha$ " model MHD equilibrium is used with $q=$ $2.2515, \hat{s}=1.1025, \alpha=0.1653$, and $\beta=0.33442 \%$. The global parameters are: $B_{0}=4.76 \mathrm{~T}, a=80.0 \mathrm{~cm}, R=245.0 \mathrm{~cm}$, and $\beta_{0}=0.96356 \%$ at the magnetic axis. Again, a carbon impurity species and a hot deuterium beam species are included in the calculations along with the background deuterium ion species and the electrons. The local parameters at $r=38 \mathrm{~cm}$ are: $n_{t:}=$ $3.9084 \times 10^{13} \mathrm{~cm}^{-3}, n_{b}=0.29774 \times 10^{13} \mathrm{~cm}^{-3}, n_{C}=0.081147 \times 10^{13} \mathrm{~cm}^{-3}$, $T_{e}=1.6294 \mathrm{keV}, T_{i}=T_{C}=1.5141 \mathrm{keV}, T_{b}=24.411 \mathrm{keV}, L_{n e} / r=4.3582$, $\eta_{e}=4.6830, \eta_{i}^{\ell}=5.5441, \eta_{b}=0.23878, r / R=0.15510, L_{n C} / L_{n e}=1.0$, and $L_{n b} / L_{n e}=0.16007$. For this case, the instability calculations are performed both in the electrostatic limit ( $\hat{\phi}$ only) and in an electromagnetic calculation ( $\hat{\phi}$ and $\hat{A}_{\|}$or $\hat{\psi}_{\|}$), with both including electron collisions but not ion collisions. In the electrostatic limit, results from the initial value calculation and the eigenvalue calculation for $\omega_{r}$ and $\gamma$ are shown versus $k_{\theta} \rho_{i}$ in Fig. 4. Again, there is good agreement between the two calculations, with $|\Delta \omega| /|\omega| \lesssim 5 \%$. The corresponding electromagnetic results are shown in Fig. 5 for fixed $k_{\theta} \rho_{\imath}=$ 0.4 , and again, there is good agreement between the two calculations, with $|\Delta \omega| /|\omega| \lesssim 6 \%$. 


\section{Conclusions}

Two completely independent computer codes which solve the same equations, namely the linearized gyrokinetic equation, along with the quasineutrality condition and Ampere's law, using the high- $n$ ballooning representation for the " $s-\alpha$ " model MHD equilibrium, have been compared. These two codes use completely different solution methods for these equations, and also use different collision operators. The initial value code uses a Lorentz collision operator, while the eigenvalue code uses a modified Krook collision operator which is designed to approximate the results of a Lorentz operator in the banana regime and the lower end of the plateau regime. After implementing several corrections in the initial value code, and after improving the Krook collision operator in the eigenvalue code, good agreement is found between the eigenfrequencies calculated by the two codes. For an artificial test case and for several cases using TFTR experimental data, the eigenfrequencies are always within 10 to $12 \%$ of one another (as measured by $|\Delta \omega| /|\omega|)$, and are usually within 5 to $6 \%$. This level of agreement gives some confidence that both codes are now working correctly. It is within the typical error of the experimental input data for density, temperature, and safety factor $q$ gradients. In particular, these comparisons verify that the implicit scheme in the initial value code is accurate, even for trapped particle effects. The instability compared here is the toroidal drift mode, destabilized by the combined effects of trapped particles and ion temperature gradients, calculated both in the electrostatic limit (with $\hat{\phi}$ only) and in the electromagnetic case (here keeping $\hat{\phi}$ and $\hat{A}_{\|}$or $\hat{\psi}_{\|}$only).

In future work, the comparison will be extended to other instabilities which can be calculated by the electromagnetic versions of both the initial value code and the eigenvalue code, namely the high- $n$ kinetic MHD ballooning mode, and possibly the high- $n$ toroidal Alfven eigenmode (TAE mode). Both calculations are susceptible of extension in various directions. The initial value calculation, in particular, can be generalized for nonlinear simulations, which is not possible for the eigenvalue calculation; this generalization is in progress. The eigenvalue code has already been extended in a number of different directions, though those extensions were not used in this comparison, in order to keep the physics in the two calculations the same. There are versions of the eigenvalue code which include $\hat{A}_{\perp}$, the perpendicu- 
lar component of the perturbed vector potential, the higher-order cylindrical magnetic drifts, an ion collision operator of the same form as the electron collision operator, the ability to use more general, numerically computed MHD equilibria, the ability to use more general, non-Maxwellian equilibrium distribution functions, in particular for the hot beam ion or hot alpha particle species, and the ability to calculate the quasi-linear particle and energy fluxes for each species associated with a linear eigenmode, as has been described already in refs. [2], [4], and [5]. Some of these extensions can also be made to the initial value code in future versions.

\section{Acknowledgments}

This work was supported by United States Department of Energy Contract Nos. DE-AC02-76-CHO-3073 and DE-FG05-80ET-53088.

\section{References}

[1] M. Kotschenreuther, et al., Proc. 14th Int. Conf. Plasma Physics and Controlled Nuclear Fusion Research, 1992, Würzburg, Germany, paper IAEA-CN-56/D1-2, IAEA, Vienna, (1993).

[2] G. Rewoldt, W.M. Tang, and R.J. Hastie, Phys. Fluids 30 (1987) 807.

[3] J.W. Connor, R.J. Hastie, and J.B. Taylor, Phys. Rev. Lett. 40 (1978) 396.

[4] G. Rewoldt, W.M. Tang, and M.S. Chance, Phys. Fluids 25 (1982) 480.

[5] G. Rewoldt and W.M. Tang, Phys. Fluids 26 (1983) 3619. 


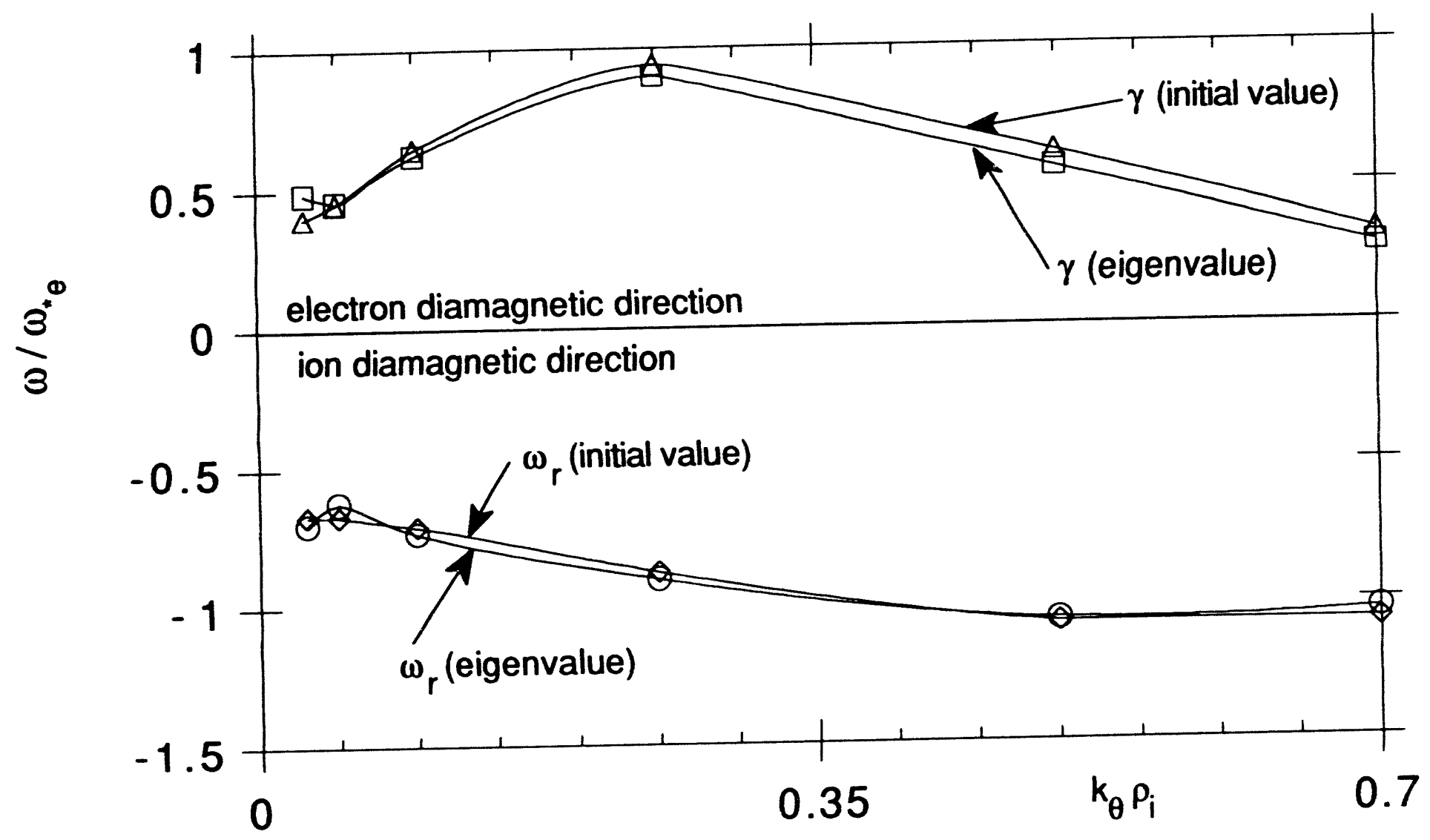

Fig. 1. Linear growth rates $\gamma$ and real frequencies $\omega_{r}$ in units of the electron diamagnetic drift frequency $\omega_{*_{e}}$ versus $k_{\theta} \rho_{i}$ from the initial value calculation and the eigenvalue calculation. Results are given for the artificial test case parameters, in the collisionless, electrostatic limit. 

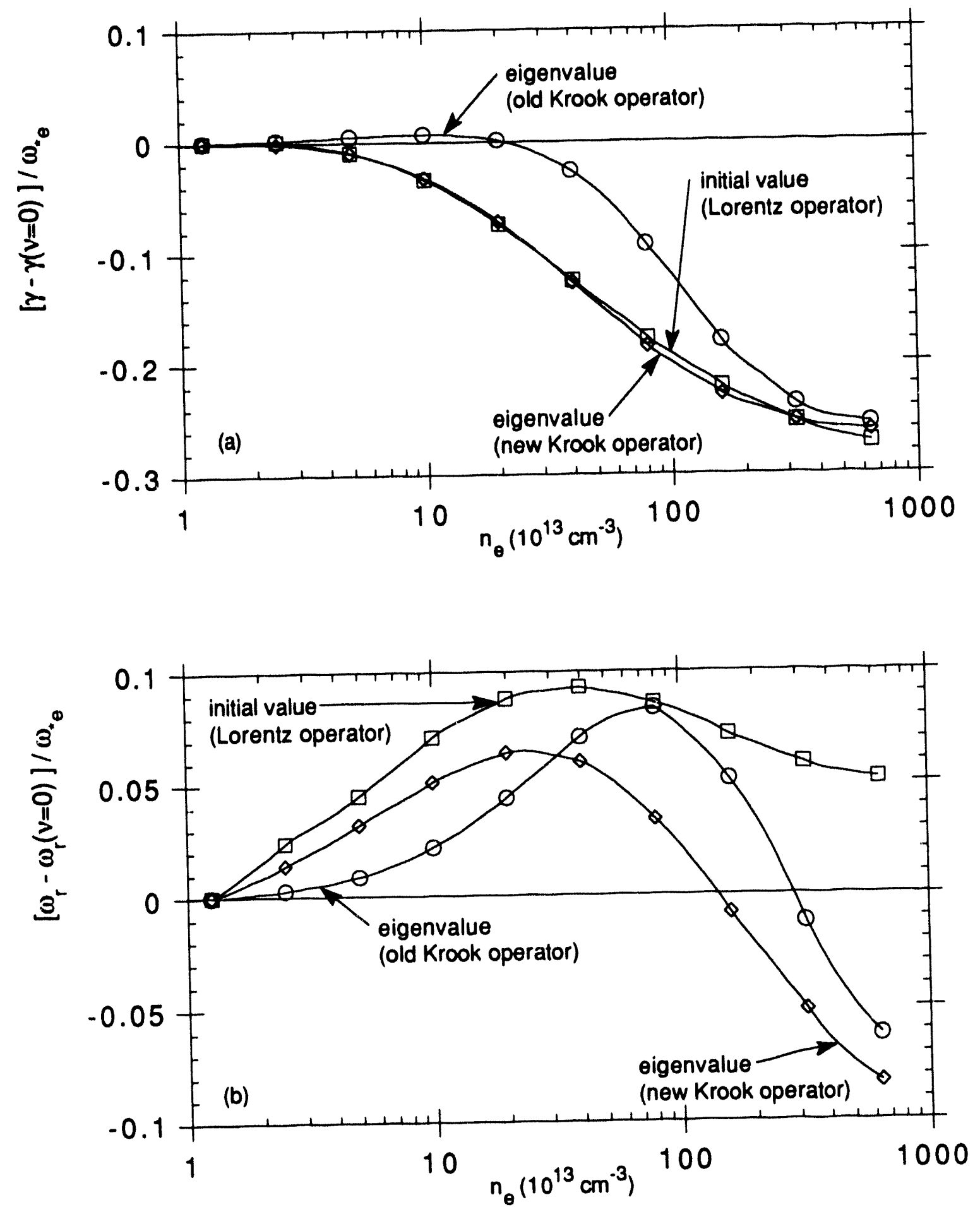

Fig. 2. Departure of $\gamma$ and $\omega_{r}$ from their respective collisionless limits, in units of $\omega_{*} e^{\prime}$ versus electron density, which is approximately proportional to the electron collision frequency $v_{e}$,from the electrostatic initial value and eigenvalue calculations. Here, the artificial test case parameters are used, with $k_{\theta} \rho_{i}=0.5$ fixed and ion collisions omitted. 


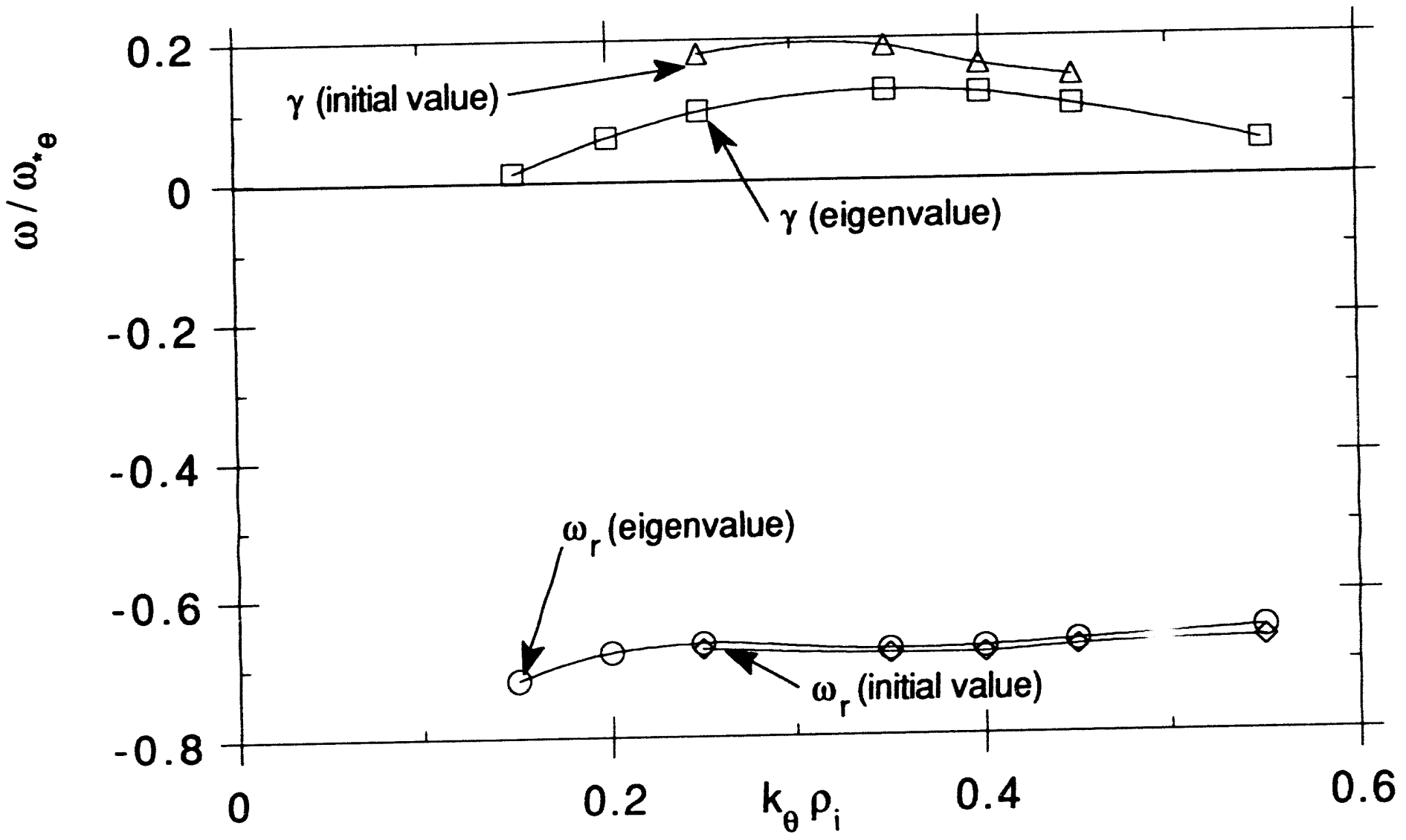

Fig. 3. Results for $\gamma$ and $\omega_{r}$ in units of $\omega_{*}$ versus $k_{\theta} \rho_{i}$, from the electrostatic initial value and eigenvalue calculations, for the TFTR L-mode shot 52504 parameters at $r=37.9 \mathrm{~cm}$. Here, a carbon impurity species and a deuterium beam species and electron collisions are included in the calculation, but ion collisions are omitted. 


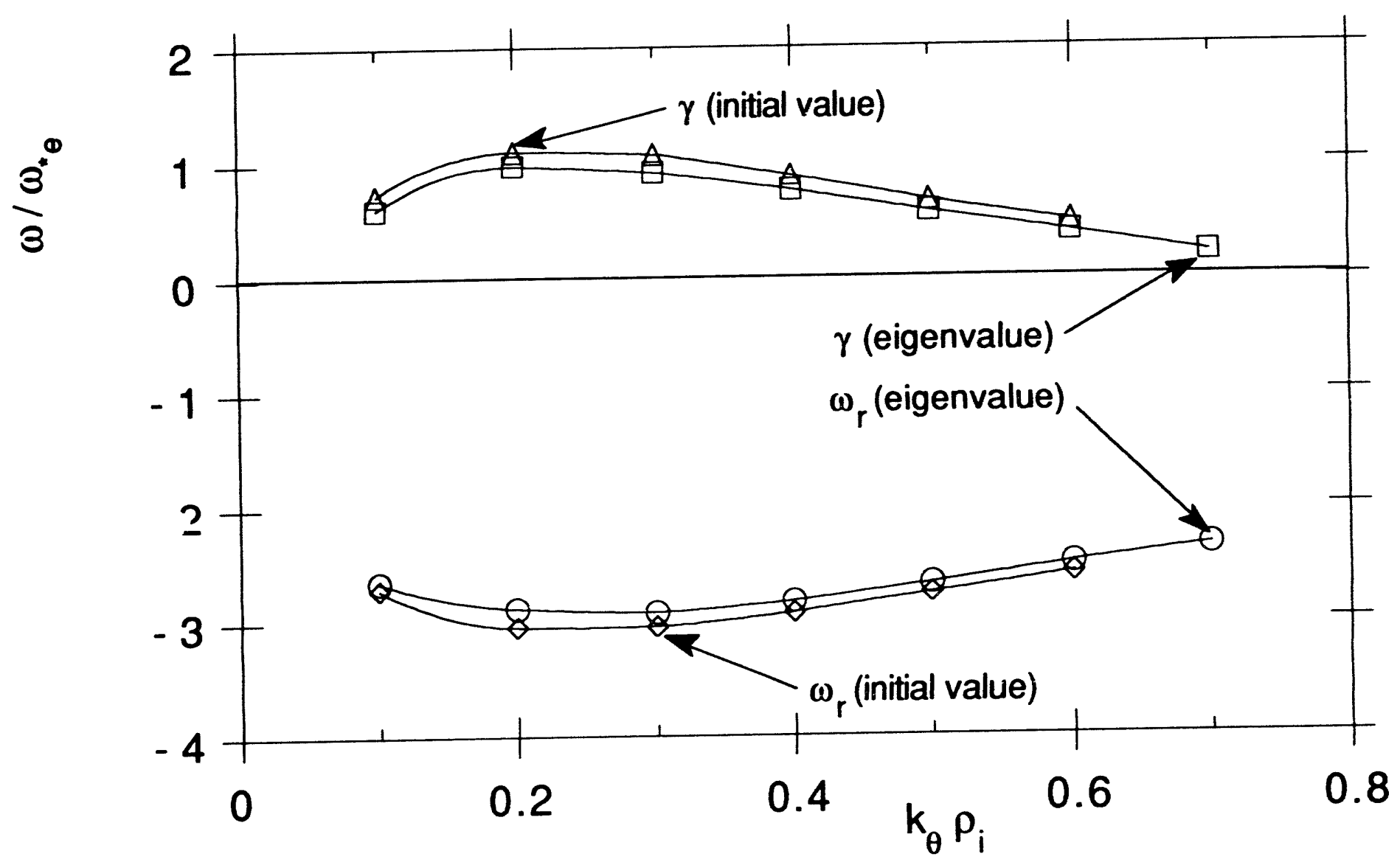

Fig. 4. Results as in Fig. 3, but for TFTR L-mode shot 49982 at $r=38 \mathrm{~cm}$. 


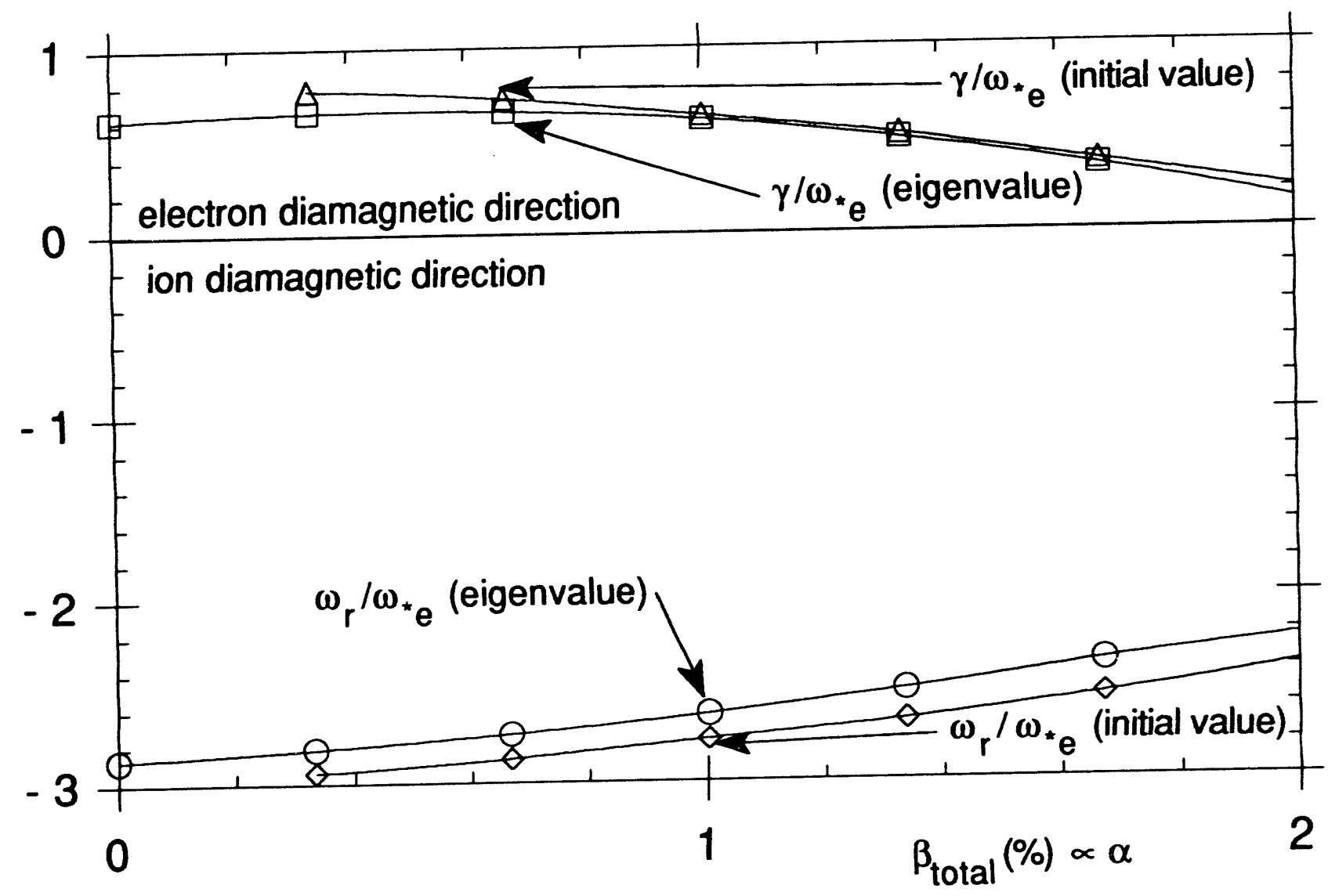

Fig. 5. Results as in Fig. 4 for $\gamma$ and $\omega_{r}$ versus the local total $\beta$, which here is proportional to the MHD equilibrium parameter $\alpha$, from the electromagnetic initial value and eigenvalue calculations, at fixed ${ }_{\theta} \rho_{i}=0.4$. 


\section{EXTERNAL DISTRIBUTION IN ADDITION TO UC-420}

Dr. F. Paoloni, Univ. of Wollongong, AUSTRALIA

Prof. M.H. Brennan, Univ. of Sydney, AUSTRALIA

Plasma Research Lab., Australian Nat. Univ., AUSTRALIA

Prof. I.R. Jones, Flinders Univ, AUSTRALIA

Prof. F. Cap, Inst. for Theoretical Physics, AUSTRIA

Prof. M. Heindler, Institut für Theoretische Physik, AUSTRIA

Prot. M. Goossens, Astronomisch Instituut, BELGIUM

Ecole Royale Militaire, Lab. de Phy. Plasmas, BELGIUM

Commission-European, DG. XII-Fusion Prog., BELGIUM

Prof. R. Bouciqué, Rijksuniversiteit Gent, BELGIUM

Dr. P.H. Sakanaka, Instituto Fisica, BRAZIL

Prof. Dr. I.C. Nascimento, Instituto Fisica, Sao Paulo, BRAZIL Instituto Nacional De Pusquisas Espaciais-INPE, BRAZIL

Documents Office, Atomic Energy of Canada Lid., CANADA

Ms. M. Morin, CCFMTokamak de Varennes, CANADA

Dr. M.P. Bachynski, MPB Technologies, Inc., CANADA

Dr. H.M. Skarsgard, Univ. of Saskatchewan, CANADA

Prof. J. Teichmann, Univ. of Montreal, CANADA

Prof. S.R. Sreenivasan, Univ. of Calgary, CANADA

Prof. T.W. Johnston, INRS-Energie, CANADA

Dr. R. Bolton, Centre canadien de fusion magnétique, CANADA

Dr. C.R. James, Univ. of Alberta, CANADA

Dr. P. Lukác, Komenského Universzita, CZECHO-SLOVAKIA

The Libranian, Culham Laboratory, ENGLAND

Library, R61, Rutherford Appleton Laboratory, ENGLAND

Mrs. S.A. Hutchinson, JET Library, ENGLAND

Dr. S.C. Shama, Univ. of South Pacific, FIJI ISLANDS

P. Mähönen, Univ. of Helsinki, FINLAND

Prof. M.N. Bussac, Ecole Polytechnique., FRANCE

C. Mouttet, Lab. de Physique des Milieux Ionisés, FRANCE

J. Radet, CEN/CADARACHE - Bat 506, FRANCE

Prof. E. Economou, Univ. of Crete, GREECE

Ms. C. Rinni, Univ. of loannina, GREECE

Preprint Library, Hungarian Academy of Soi., HUNGARY

Dr. B. DasGupta, Saha Inst. of Nuclear Physics, INDIA

Dr. P. Kaw, Inst. for Plasma Research, INDIA

Dr. P. Rosenau, Israel Inst. of Technology, ISRAEL

Librarian, International Center for Theo Physics, ITALY

Miss C. De Palo, Associazione EURATOM-ENEA, ITALY

Dr. G. Grosso, Istituto di Fisica del Plasma, ITALY

Prof. G. Rostangni, Istituto Gas Ionizzati Del Cnr, ITALY
Dr. H. Yamato, Toshiba Res \& Devel Center, JAPAN

Prof. I. Kawakami, Hiroshima Univ., JAPAN

Prof. K. Nishikawa, Hiroshima Univ., JAPAN

Librarian, Naka Fusion Research Establishment, JAERI, JAPAN

Director, Japan Atomic Energy Research Inst., JAPAN

Prof. S. Itoh, Kyushu Univ., JAPAN

Research Info. Ctr., National Instit. for Fusion Science, JAPAN

Prof. S. Tanaka, Kyoto Univ., JAPAN

Libray, Kyoto Univ., JAPAN

Prof. N. Inove, Univ. of Tokyo, JAPAN

Secretary, Plasma Section, Electrotechnical Lab., JAPAN

S. Mori, Technical Advisor, JAERI, JAPAN

Dr. O. Mitarai, Kumamoto Inst. of Technology, JAPAN

Dr. G.S. Lee, Korea Basic Sci. Ctr., KOREA

J. Hyeon-Sook, Korea Atomic Energy Research Inst., KOREA

D.I. Choi, The Korea Adv. Inst. of Sci. \& Tech., KOREA

Prof. B.S. Liley, Univ. of Waikato, NEW ZEALAND

Inst of Physics, Chinese Acad Sci PEOPLE'S REP. OF CHINA Library, Inst. of Plasma Physics, PEOPLE'S REP. OF CHINA Tsinghua Univ. Library, PEOPLE'S REPUBLIC OF CHINA 2. Li, S.W. Inst Physics, PEOPLE'S REPUBLIC OF CHINA Prof. J.A.C. Cabral, Instituto Superior Tecnico, PORTUGAL Prot. M.A. Hellberg. Univ. of Natal, S. AFRICA Prot. D.E. Kim, Pohang Inst. of Sci. \& Tech., SO. KOREA

Prot. C.I.E.M.A.T. Fusion Division Library, SPAIN

Dr. L. Stenflo, Univ. of UMEA, SWEDEN

Library, Royal Inst. of Technology, SWEDEN

Prof. H. Wilhelmson, Chalmers Univ. of Tech., SWEDEN

Centre Phys. Des Plasmas, Ecole Polytech, SWITZERLAND

Bibliotheek, Inst. Voor Plasma-Fysica, THE NETHERLANDS

Asst. Prol. Dr. S. Cakir, Middle East Tech. Univ., TURKEY

Dr. V.A. Glukhikh,Sci. Res. Inst. Electrophys.I Apparatus, USSR

Dr. D.D. Ryutov, Siberian Branch of Academy of Sci., USSR

Dr. G.A. Eliseev, I.V. Kurchatov Inst, USSR

Librarian. The Ukr.SSR Academy of Sciences, USSR

Dr. L.M. Kovrizhnykh, Inst. of General Physics, USSR

Kernforschungsanlage $\mathrm{GmbH}$, Zentralbibliothek, W. GERMANY Bibliothek, Inst. Für Plasmaforschung, W. GERMANY

Prof. K. Schindler, Ruhr-Universitát Bochum, W. GERMANY Dr. F. Wagner, (ASDEX), Max-Planck-Institut, W. GERMANY Librarian, Max-Planck-Institut, W. GERMANY 
11
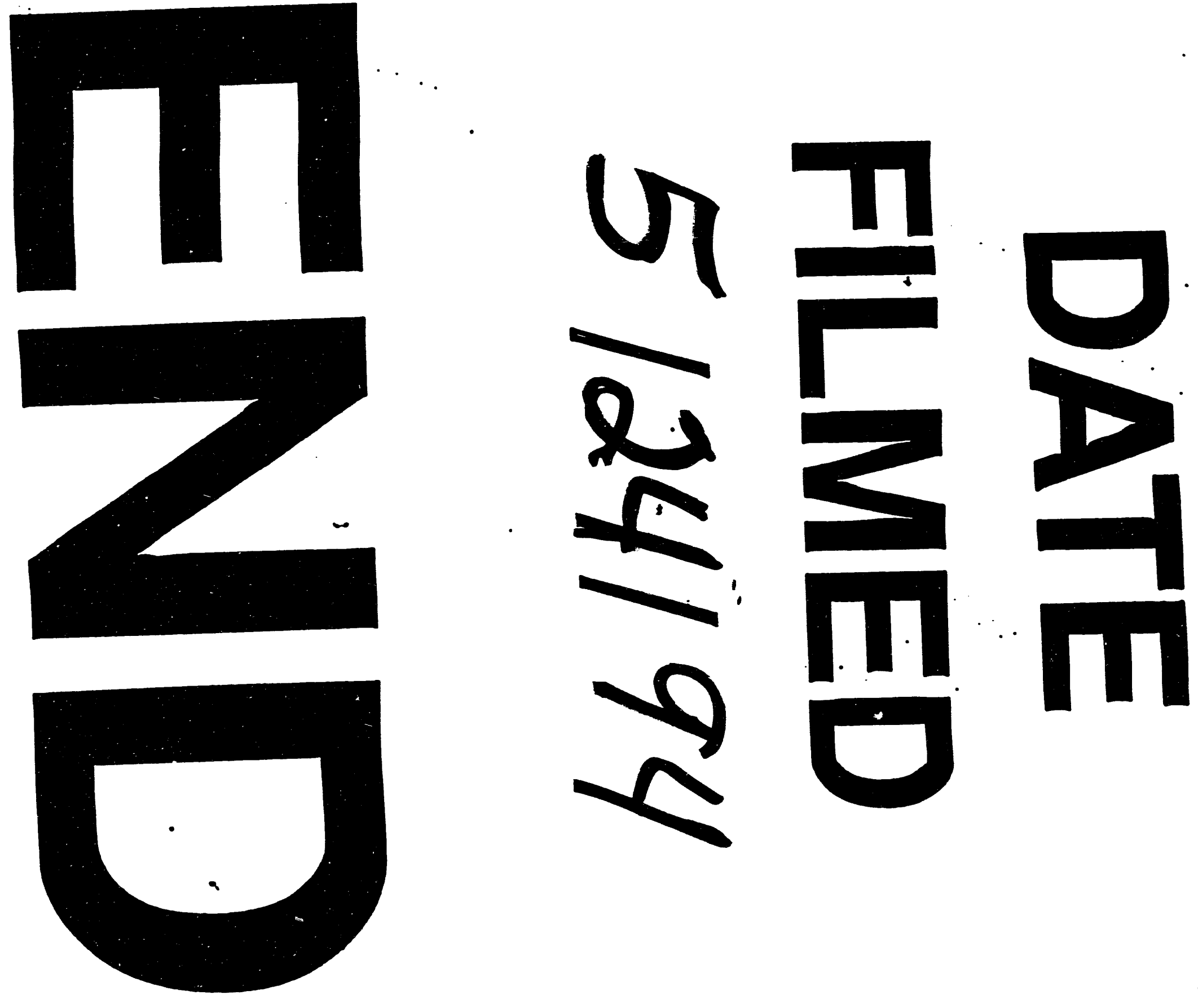


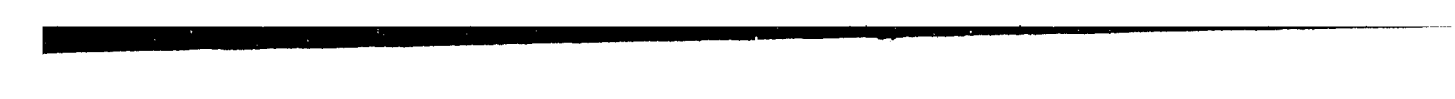

$\mid$

$+$ 